\title{
Diode Pumped Solid State Lasers for Surface Microtexture
}

\author{
Mool C. Gupta, Longteng Wang, Christian Rothenbach and Keye Sun \\ Department of Electrical \& Computer Engineering, University of Virginia, Charlottesville, VA 22904 \\ E-mail:mgupta@virginia.edu
}

\begin{abstract}
Surface microtexture has been observed under nanosecond pulsed laser irradiation of silicon and metals. Diode pumped solid state lasers with pulse width in the nanosecond regime can provide a low cost method for fabrication of large area microtextured surfaces. Results are presented on control of microtexture height using laser processing parameters and mechanism of microtexture formation is described. Microtexture height can be controlled from less than a micron to tens of microns with multiple laser pulse irradiation. The reflectivity of the microtextured surface in the visible spectrum was reduced to lower than $5 \%$.
\end{abstract}

DOI:10.2961/jlmn.2013.02.0002

Keywords: lasers, microtexture, solid state lasers, nanosecond, silicon, metals

\section{Introduction}

Currently there is a significant interest in understanding the mechanism of microtexture formation under femtosecond laser irradiation [1-5]. Several research groups have reported results on microtexture fabrication, characterization, mechanism of formation and their applications with primary focus using femtosecond and excimer lasers. Ultrafast laser generated microtextured surfaces have applications in photovoltaics [6, 7], photodetectors [8, 9], water repellant surfaces (superhydrophobic) [10] etc. Few research papers have reported similar microtexture formation in silicon using excimer laser [11]. However, the difficulty with femtosecond and excimer lasers for surface microtexture has been that it is not practical to produce large area in a cost effective manner.

We have carried out experiments using high repetition rate diode pumped solid state lasers which can provide a low cost method for fabrication of large area microtextured surfaces. Diode pumped solid state lasers with pulse width at around $10 \mathrm{~ns}$ are well suited for surface microtexture

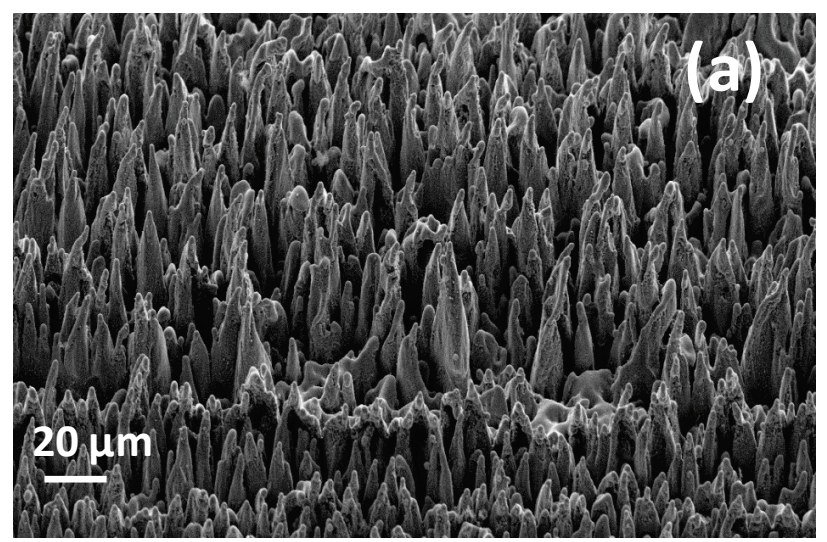

formation. The reported laser pulse width for microtexture formation ranges from about hundred femtosecond (for femtosecond lasers) to about $10 \mathrm{~ns}$ (for excimer lasers) as longer pulse width causes significant thermal diffusion and inhibits the formation of microtextures. When laser pulse widths are longer than about $10 \mathrm{~ns}$, surface microtexture formation is not reported. The laser induced microtexture has been obtained on a variety of surfaces including dielectrics, semiconductors, metals and polymers [11-14]. In this paper we provide results on dependence of microtexture height on nanosecond pulsed laser processing parameters and mechanism of microtexture formation is described.

\section{Experimental}

The surface microtexture experiments were carried out using three lasers: a diode pumped solid state laser from Quantel Lasers (model Ultra 50), fiber laser from IPG Photonics (model YLP-G-10) and Nitrogen laser from Stanford Research Systems (model NL-100). Samples were placed in a vacuum chamber mounted on a high-precision,

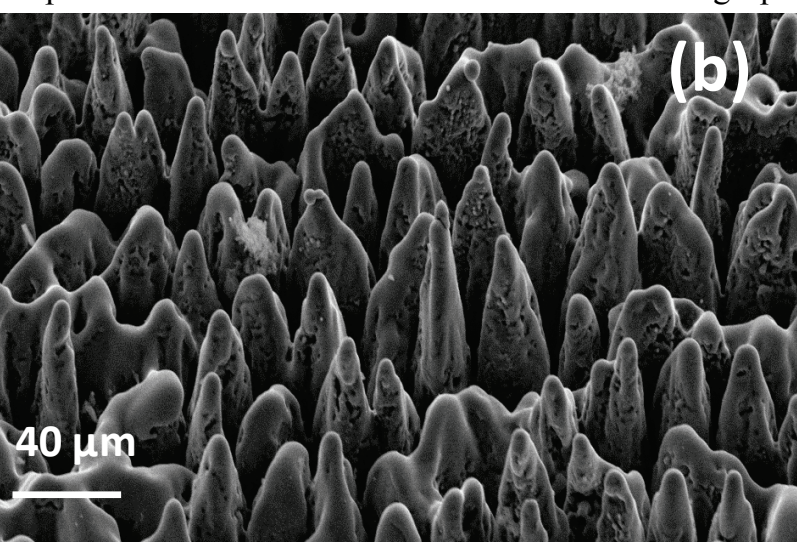

Fig. 1. SEM micrographs of microtextured Si sample. (a) Scan speed $=0.08 \mathrm{~mm} / \mathrm{s}$, equivalent to about 100 overlapping laser pulses at each point. (b) Higher magnification image of (a). 


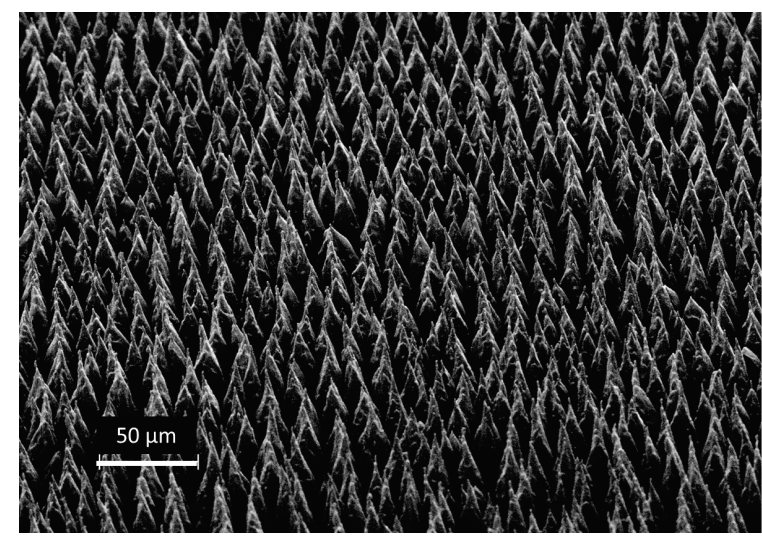

Fig. 2. SEM micrographs of microtextured Ge sample at a $0.05 \mathrm{~mm} / \mathrm{s}$ scan speed, equivalent to 320 pulses.

computer controlled X-Y stage (Aerotech, model: ATS 125100 ) in order to make more uniform microtextured large areas. The experiments were carried out in vacuum (base pressure $1 \mathrm{mTorr}$ ) as well as in air at atmospheric conditions.

Using the Quantel laser, microtexturing experiments were carried out on $1 \mathrm{~cm}^{2}$ silicon and germanium samples, and $6.25 \mathrm{~cm}^{2}$ stainless steel samples. Samples were irradiated with $32 \mathrm{~mJ}$ laser pulses of $532 \mathrm{~nm}$ wavelength, 8 ns pulse duration and repetition rate of $20 \mathrm{~Hz}$. For $\mathrm{Si}$ microtexturing experiments, the laser beam was focused using a $125 \mathrm{~mm}$ focal length lens to a spot size of about 1 $1.5 \mathrm{~mm}$. For experiments with $\mathrm{Ge}$, a $400 \mathrm{~mm}$ lens was used

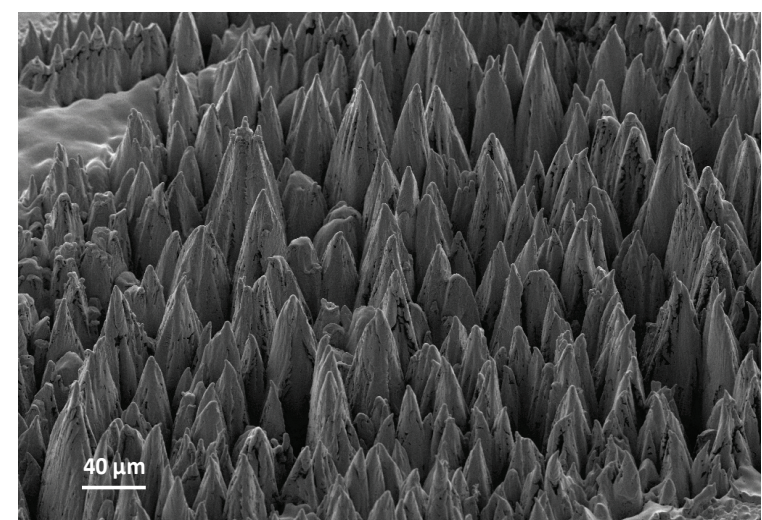

Fig. 3. SEM micrograph of microtextured stainless steel sample after 18,000 pulses.

to focus the laser beam to allow for large area surface microtexturing and the beam was focused to a size of 1.6 $\mathrm{mm}$. For stainless steel experiments, the laser beam was focused using a $125 \mathrm{~mm}$ lens to a spot size of $600-800 \mu \mathrm{m}$ as well.

IPG Photonics YLP-G-10 laser is a nanosecond fiber laser with pulse energy of $16 \mu \mathrm{J}$ and wavelength of $532 \mathrm{~nm}$. The repetition rate can be varied from $20 \mathrm{kHz}$ to $600 \mathrm{kHz}$. The pulse width is 1 ns. The laser was ideal for microtexturing of silicon and germanium because of the relatively shallow absorption depth of silicon and germanium at $532 \mathrm{~nm}$ wavelength and also its short pulse
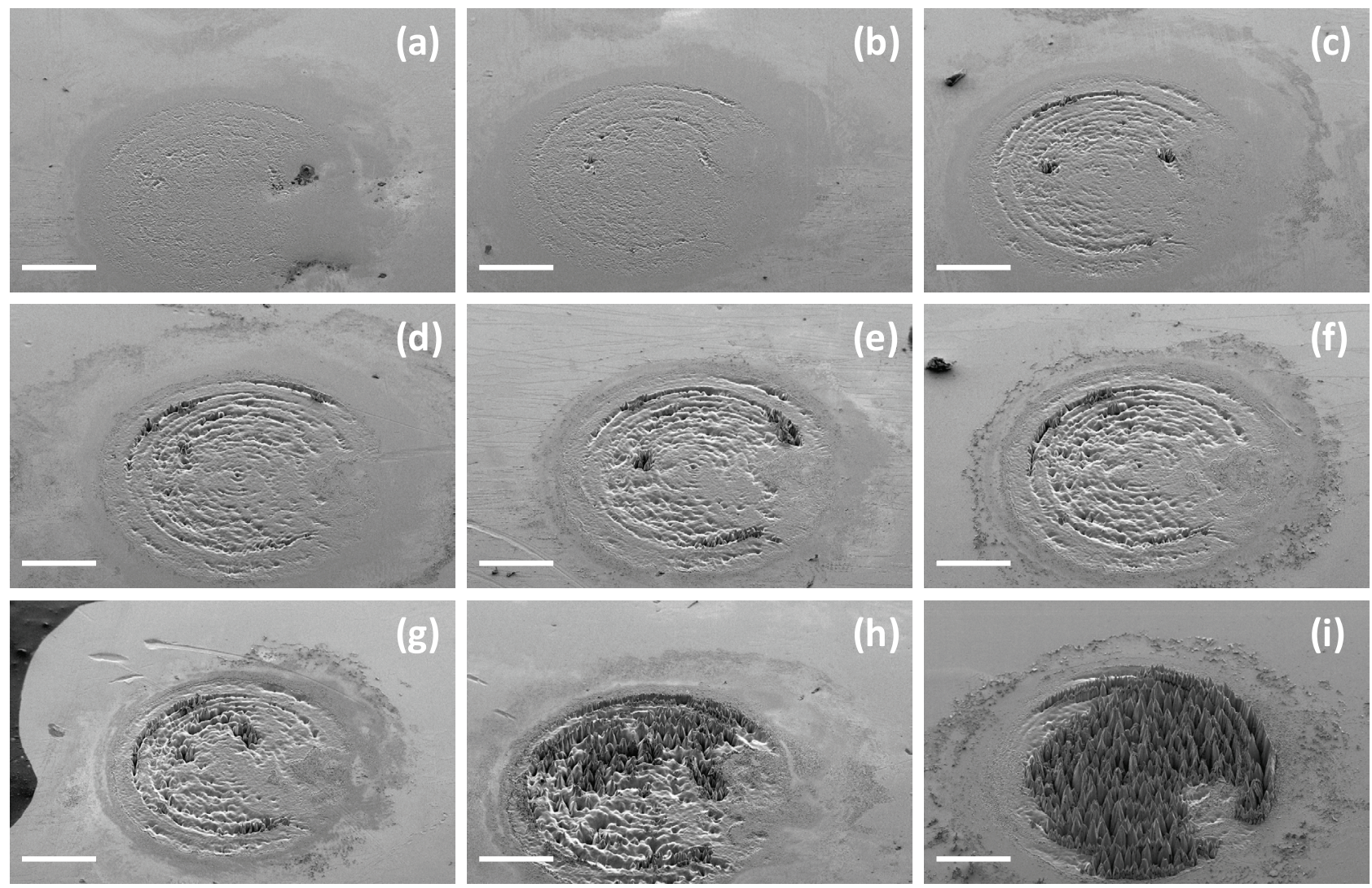

Fig. 4. SEM micrographs of microtexture evolution on stainless steel at (a) 200, (b) 400, (c) 2,000, (d) 3,000, (e) 5,000, (f) 6,000, (g) 9,000, (h) 12,000 and (i) 18,000 pulses. The scale bar in (a)-(i) is $200 \mu \mathrm{m}$. 


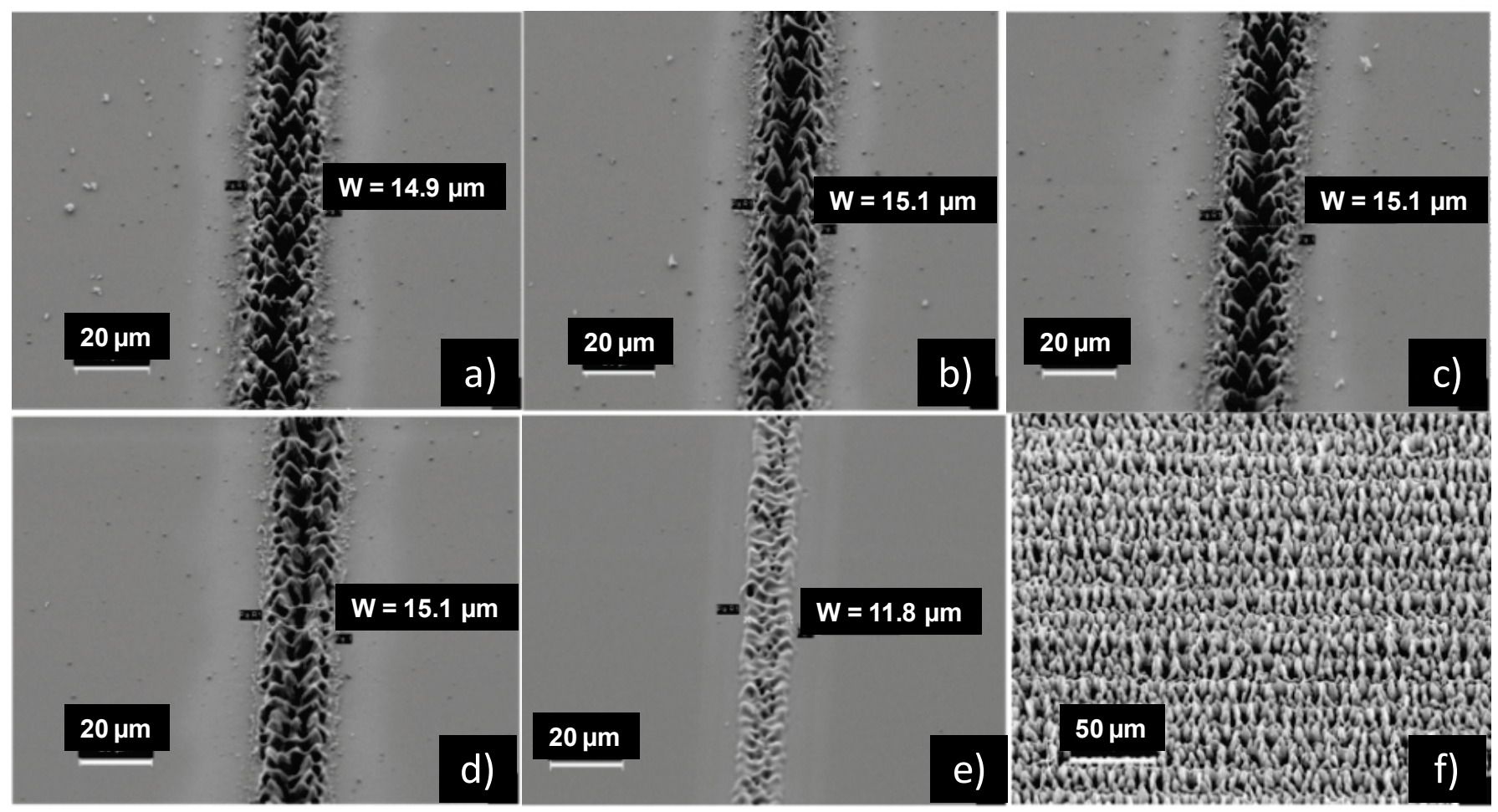

Fig. 5. SEM images of varying laser scan speeds with laser energy density kept at $1.5 \mathrm{~J} / \mathrm{cm}^{2}$ (a) $5 \mathrm{~mm} / \mathrm{s}(80$ pulses $)(\mathrm{b}) 6 \mathrm{~mm} / \mathrm{s}(66$ pulses) (c) $8 \mathrm{~mm} / \mathrm{s}$ (50 pulses) (d) $12 \mathrm{~mm} / \mathrm{s}$ (33 pulses) (e) $25 \mathrm{~mm} / \mathrm{s}$ (16 pulses) (f) large area microtextured Si generated by raster scanning with laser scanning speed of $5 \mathrm{~mm} / \mathrm{s}$ and spacing of $12 \mu \mathrm{m}$ between scanning lines.

width. Experiments were carried out on Si samples.

The nitrogen laser from Stanford Research Systems has a repetition rate of $20 \mathrm{~Hz}, 170 \mu \mathrm{J}$ pulse energy and $337 \mathrm{~nm}$ wavelength. Experiments were carried out on Si samples, as recently reported [12].

The surface morphology of the laser microtextured samples was investigated by a Zeiss SUPRA 40 scanning electron microscope (SEM). The height of microtextures was measured by the calibrated tool on the cross-section SEM image with accuracy of $0.5 \mu \mathrm{m}$. Total Integrated reflection as a function of wavelength was measured using a 2-inch integrating sphere (STELLARNET Inc) at an incident angle of 35 degrees.

The appropriate laser conditions for microtexturing were found by tuning the laser energy from low to high. The threshold of microtexturing generally resides between melting and ablation. Laser scanning speed is adjusted to have different number of overlapping laser pulses at each point on sample surface. This could affect the surface morphology by changing the height of microtextures.

\section{Results and Discussion}

\section{A. Microtexture formation studies using diode pumped} solid state laser

The experiments carried out on silicon, germanium and stainless steel described in this section were performed with the Quantel Ultra 50 solid state laser. Si (100) samples were microtextured and Fig. 1 shows SEM micrographs of these samples. Texture height was measured to be within the range of 20 to $30 \mu \mathrm{m}$. Fig. 2 shows SEM micrographs of the microtextured Ge samples. The structure was formed at 320 pulses per spot on average with an energy density of 1.6 $\mathrm{J} / \mathrm{cm}^{2}$. Texture height was measured to be less than $20 \mu \mathrm{m}$. It should be pointed out that laser microtexturing of silicon and germanium was also done in air or a shielding gas such as Argon. Similar surface morphologies were observed with more particles and debris on surface probably induced by oxidation.

Fig. 3 shows a stainless steel sample that was microtextured using 18,000 laser pulses. Microtexture height varied from 60 to $120 \mu \mathrm{m}$. Due to the higher melting threshold of stainless steel $\left(\sim 1 \mathrm{~J} / \mathrm{cm}^{2}\right)$ as compared to $\mathrm{Si}$ $\left(\sim 0.6-1 \mathrm{~J} / \mathrm{cm}^{2}\right)$ and $\mathrm{Ge}\left(\sim 0.5 \mathrm{~J} / \mathrm{cm}^{2}\right)$, to generate the surface microtexture, more laser pulses were required. Even at very low stage scanning speeds of $0.01 \mathrm{~mm} / \mathrm{s}$, several hundred laser scans were required before microtexture formation could be observed. Instead, the laser beam was allowed to dwell on the sample in order to obtain a larger number of pulses on the same area.

In order to study the mechanism of microtexture evolution in stainless steel, we carried out dwell spot experiments by varying the number of laser pulses impinging on a given spot on the sample. This evolution is show in Fig. 4. At 200 laser pulses, the laser energy is sufficient to start melting the exposed area. Ripples begin to form in the melt. The formation of the ripples in turn starts forming conical structures. At 2,000 laser pulses, it is possible to see the conical structures protruding in some 


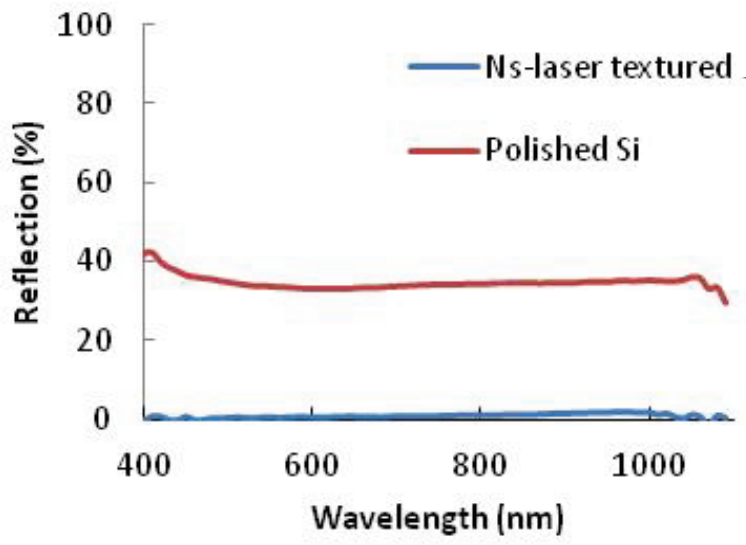

Fig. 6. Reflection of nanosecond laser microtextured crystalline silicon from sample shown in Fig. 5(f) and of polished samples.

parts of the laser exposed area. With increase in laser pulses, more conical structures start appearing in areas where the beam has a higher power. As more laser pulses are provided, the number of ripples and cones increases until most of the area is microtextured at 18,000 laser pulses. The observed ripples and general shape of the formed spots are due to the non-uniformity of the laser beam profile. Similar microtexture growth has been observed in $\mathrm{Si}$ using a nanosecond nitrogen laser [4].

\section{B. Microtexture formation studies using fiber laser}

The experiments performed on silicon and germanium as discussed in this section were carried out using an IPG Photonics YLP-G-10 fiber laser. The microtexture morphology can be controlled by the laser scan parameters. By varying the scan speed, the height of the microtexture can be varied. The results are shown in Fig. 5. The laser conditions in this experiments resulted in a spot size of about $20 \mu \mathrm{m}$. As can be seen, the mixcrotexture height decreased with increasing laser scan speed. Also shown in Fig. 5 is the SEM image of large area microtextured crystalline silicon.

The optical reflection of crystalline silicon after microtexturing is shown in Fig. 6. The reflection of polished silicon is also shown in the same figure as a comparison. Such measurement was carried out for the sample shown in Fig 5(f). As can be seen, the microtextured silicon has a reflection below $5 \%$, which is much lower than polished silicon. Furthermore, the reflection of the laser microtextured silicon is much less dependent on wavelength compared to polished silicon. Unlike chemical texturing, laser microtexturing doesn't distinguish between single crystalline and multi-crystalline silicon. Lasers can microtexture different kinds of silicon as well as single crystalline silicon. The morphologies of laser microtextured multi-crystalline and $50 \mu \mathrm{m}$ thin silicon wafer are shown in Fig. 7. The cross section of laser microtextured thick silicon wafer was generated by mechanical cleaving. The laser conditions for figure 7(a) were the same as in the sample of figure 5(f). As for the thin Si wafer in figure 7(b), laser scanning speed was $10 \mathrm{~mm} / \mathrm{s}$ equivalent to 40 overlapping laser pulses on each point.

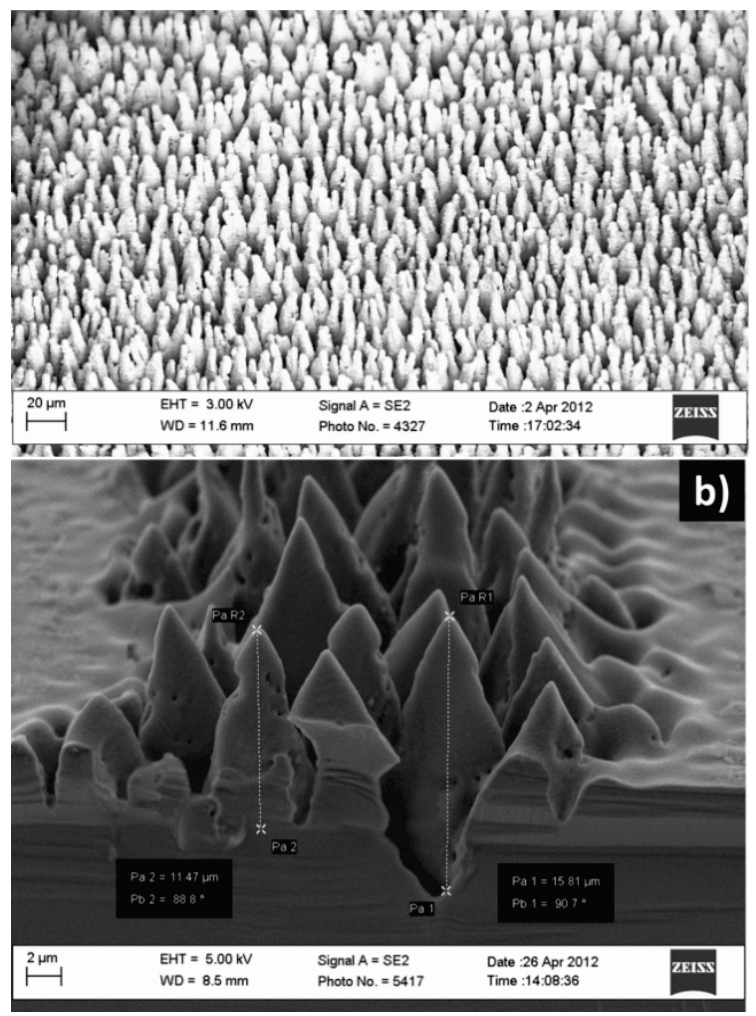

Fig. 7. a) ns-laser textured multi-crystalline silicon for 80 pulses, b) cross section of ns-laser textured thin silicon wafer for 40 pulses.

As mentioned, the fiber nanosecond laser was for microtexturing of germanium. The morphology is shown in Fig. 8. The structures were formed using $1.3 \mathrm{~J} / \mathrm{cm}^{2}$ laser energy density at a repetition rate of $20 \mathrm{kHz}$. The average number of pulses per spot is about 50 . The optical reflection of the laser microtextured surface (shown in Fig. 8) is given in Fig. 9. Similar to the silicon case, the reflection was reduced greatly after laser microtexturing.

\section{Microtexture formation studies using nitrogen laser}

Recently [4] we have reported that a low cost nitrogen laser can be used for surface microtexture applications. The microtexture observed with nitrogen laser appears to be similar to the diode pumped solid state and fiber laser. The nitrogen laser provides a very low-cost method for microtexturing as laser cost was about $\$ 8,000$. However, the laser has a low repetition rate of $20 \mathrm{~Hz}$ which limits the microtexturing processing speed. At a wavelength of 337 $\mathrm{nm}$ for the nitrogen laser, the absorption depth in $\mathrm{Si}$ is about $10 \mathrm{~nm}$, allowing generation of high energy density.

\section{Comparison between femtosecond and nanosecond laser induced microtexture}

We have demonstrated that low cost nanosecond pulse width solid state lasers can be successfully used to form surface microtextures, which avoids the use of expensive femtosecond lasers. Microtexture height can be varied from less than micron to tens of microns. A comparison of results from various lasers is presented in Table $\mathrm{I}$. 


\begin{tabular}{|c|c|c|c|c|}
\hline & Femtosecond laser & $\begin{array}{c}\text { Quantel diode } \\
\text { pumped solid state } \\
\text { laser }\end{array}$ & IPG Fiber Laser & Nitrogen laser \\
\hline Maximum pulse energy & $1 \mathrm{~mJ}$ & $32 \mathrm{~mJ}$ & $16 \mu \mathrm{J}$ & $170 \mu \mathrm{J}$ \\
\hline Pulse duration & $40 \mathrm{fs}$ & $8 \mathrm{~ns}$ & $1 \mathrm{~ns}$ & $3.5 \mathrm{~ns}$ \\
\hline Wavelength & $800 \mathrm{~nm}$ & $532 \mathrm{~nm}$ & $532 \mathrm{~nm}$ & $337 \mathrm{~nm}$ \\
\hline Absorption depth in $\mathrm{Si}$ & $\sim 10 \mu \mathrm{m}$ & $\sim 1 \mu \mathrm{m}$ & $\sim 1 \mu \mathrm{m}$ & $\sim 10 \mathrm{~nm}$ \\
\hline Beam profile & Gaussian & $\begin{array}{l}\text { Essentially Gaussian } \\
\text { (with some non- } \\
\text { uniformity) }\end{array}$ & Gaussian & Rectangular \\
\hline $\begin{array}{c}\text { Energy density for } \\
\text { microtexturing } \\
\text {-Si } \\
- \text { Ge } \\
\text {-Steel } \\
\end{array}$ & $\begin{array}{l}1 \mathrm{~J} / \mathrm{cm}^{2} \\
0.6 \mathrm{~J} / \mathrm{cm}^{2} \\
1.2 \mathrm{~J} / \mathrm{cm}^{2} \\
\end{array}$ & $\begin{array}{l}1.5 \mathrm{~J} / \mathrm{cm}^{2} \\
1.3 \mathrm{~J} / \mathrm{cm}^{2} \\
3.3 \mathrm{~J} / \mathrm{cm}^{2} \\
\end{array}$ & $\begin{array}{l}1.5 \mathrm{~J} / \mathrm{cm}^{2} \\
1.3 \mathrm{~J} / \mathrm{cm}^{2} \\
1.3 \mathrm{~J} / \mathrm{cm}^{2}\end{array}$ & $\begin{array}{c}2 \mathrm{~J} / \mathrm{cm}^{2} \\
\text { Data not available } \\
\text { Data not available }\end{array}$ \\
\hline $\begin{array}{c}\text { Number of pulses for } \mathrm{Si} \\
\text { microtexturing }\end{array}$ & $5-50$ & $50-100$ & $50-100$ & $50-100$ \\
\hline Nanoparticles on surface & Large number & No & No & No \\
\hline $\begin{array}{l}\text { Morphology for } \mathrm{Si} \\
\text { microtextures }\end{array}$ & Sharp cones & Rounded tips & Rounded tips & High sharp cones \\
\hline Crystalline structure & Amorphous layer & Crystalline & Crystalline & Crystalline \\
\hline Reflection & $<5 \%$ & $<5 \%$ & $<5 \%$ & $<5 \%$ \\
\hline Beyond band absorption & Observed & Data not available & Observed & Data not available \\
\hline Fabrication speed & Low & Medium & High & Low \\
\hline Shielding gas & Not required & Not required & Not required & Not required \\
\hline Footprint & Large & Small & Small & Small \\
\hline Cost & High & Medium & Medium & Low \\
\hline
\end{tabular}




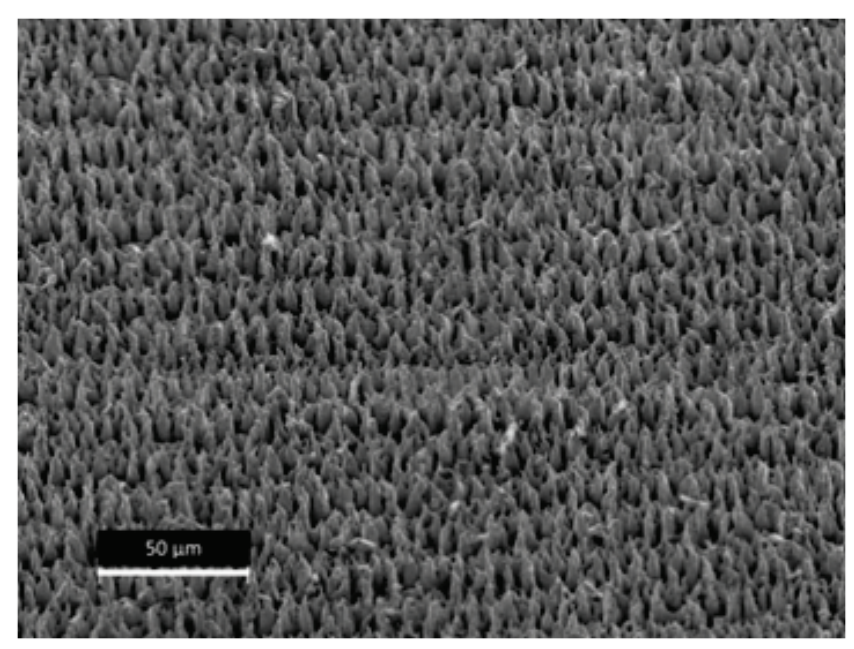

Fig. 8. Ge microtextured with nanosecond fiber laser, with energy density of $1.35 \mathrm{~J} / \mathrm{cm}^{2}$ and 50 pulses.

The mechanism of surface microtexture formation is discussed in various literatures [15]. After the laser pulse is absorbed by the material, the temperature rises, melting, vaporization and ablation occur. During the cooling process, the molten materials rearrange themselves to create certain roughness patterns. Additional laser pulses generate more roughness. Because of the shadowing effects of the original roughness created by the previous pulses, the textured features are deepened and finally lead to surface microtexture. The microtexture generation occurs through thermo-mechanical process. Multiple laser pulses are required to achieve certain microtexture height. The number of pulses required to form microtexture is dependent on the laser power, melting threshold of the material and laser wavelength.

Surface microtexture can be formed both by femtosecond and nanosecond lasers $[16,4]$, but they differ in several ways. The forming mechanisms are different for femtosecond and nanosecond lasers. For femtosecond lasers, the magnitude of the electric field associated with the extremely short laser pulses is strong that it makes the interaction highly nonlinear. The nonlinear interaction between the laser pulses and material decreases the absorption depth. Thus the volume into which the energy deposited is small, which generates the high energy density in the material. On the other hand, the thermalization of hot electrons with crystal lattice takes about 1 to $10 \mathrm{ps}$ which is much longer than the femtosecond laser pulses. A highdensity electron-hole plasma is formed at the surface after the irradiation of the laser pulses [17]. Depending on the energy of the laser pulse, melting, ablation or non-thermal melting [18] could happen and surface roughness is created due to these processes. For nanosecond lasers, since the pulse width is much longer than the electron thermalization time, the roughness forming mechanism is more of melting, flow and resolidification and is believed to be thermo-

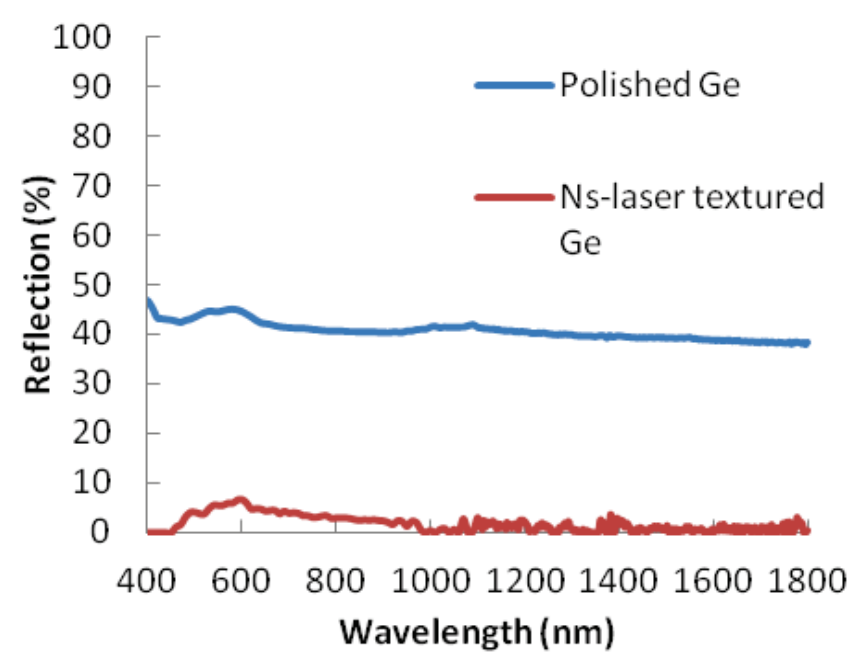

Fig. 9. Reflection of polished and ns-laser microtextured Ge.

mechanically dominated. Due to the different forming mechanisms, the number of pulses to form the microtextures is much less for femtosecond laser than nanosecond laser. Also attributed to the different forming mechanisms, the resulting properties are different for femtosecond and nanosecond laser-induced surface microtextures [19]. The surface of microtextures formed by femtosecond laser is covered by nanoparticles while the nanosecond laser-formed microtextures have much smoother surfaces. Also, most of the femtosecond laser-formed microtextures are below the original surface while nanosecond laser-formed microtextures are both above and below the original surface. This indicates that femtosecond laser forming mechanism is ablation-dominated while the nanosecond laser-induced microtextures are formed by flow of molten material. For the electronic properties, femtosecond laser-formed microtextures have a crystalline core covered by an amorphous shell while the nanosecond laser-formed microtextures are nearly single crystalline. For optical properties, both femtosecond and nanosecond laser-formed microtextures have near-unity absorption for the photon energy above band gap. Interestingly, both microtextures show certain absorption in the beyond band gap spectrum which is coming from the defects and Urbach band tails of amorphous shell formed during the laser microtexturing [20].

\section{Conclusions}

Experiments carried out using Quantel laser (model: Ultra 50), the IPG fiber laser (model: YLP-G-10) and Stanford Research Systems (model: NL-100) showed that it was possible to microtexture the surfaces of semiconductor substrates such as $\mathrm{Si}, \mathrm{Ge}$ and stainless steel metallic alloys. We have also shown that fiber lasers with very high repetition rates of over $600 \mathrm{kHz}$ can be used for large area surface microtexture. A comparison of laser based microtexture properties with nanosecond pulse width laser 
has been made. While microtexture formation has been demonstrated in air, to prevent oxidation, use of gas assisted microtexturing process can be employed.

\section{Acknowledgments}

We thank, DARPA-MTO, NSF I/UCRC and NASA Langley Professor program for their financial support.

\section{References}

[1] T. Her, R. J. Finlay, C. Wu, S. Deliwala and E. Mazur, Appl. Phys. Lett., 73 (1998) 1673.

[2] B. K. Nayak and M. C. Gupta, Materials Research Society Symposium Proceedings, 850 (2005) MM1.8.1.

[3] D. H. Lowndes, J. D. Fowlkes and A. J. Pedraza, Appl. Surf. Sci., 154 (2000) 647.

[4] B. K. Nayak and M. C. Gupta, Applied Optics, 51(2012) 114.

[5] B. K. Nayak and M. C. Gupta, Optics \& Lasers in Engineering, 48 (2010) 966.

[6] V. V. Iyengar, B. K. Nayak and M. C. Gupta, Solar Energy Materials and Solar Cells, 94 (2010) 225.

[7] B. K. Nayak, V. V. Iyengar and M. C. Gupta, Progress in Photovoltaics Research and Applications, 19 (2011) 631.

[8] Z. Huang, J. E. Carey, M. Liu, X. Guo, E. Mazur and J. C. Campbell, Appl. Phys. Lett., 89 (2006) 033506.
[9] Z. Li, B. K. Nayak, V. V. Iyengar, D. McIntosh, Q. Zhou, M. C. Gupta and J. C. Campbell, Appl. Optics, 50 (2011) 2508.

[10] P. O. Caffrey, B. K. Nayak and M. C. Gupta, Applied Optics, 51 (2012) 604.

[11] A. J. Pedraza, J. D. Fowlkes, and D. H. Lowndes, Appl. Phys. Lett., 74 (1999) 2322.

[12] X. Wang, K. Kato, K. Adach, K. Zizawa, Tribology International, 34 (2001) 703.

[13] B. K. Nayak, M. C. Gupta, K. W. Kolasinski, Appl. Phys. A., 90 (2008) 399.

[14] P. E. Dyer, S. D. Jenkins, J. Sidhu, Appl. Phys. Lett., 52 (1988) 1880.

[15] B. K. Nayak, M. C. Gupta, Optics and Lasers in Engineering, 48 (2010) 966.

[16] B. K. Nayak, M. C. Gupta, K. W. Kolasinski, Nanotechnology, 18 (2007) 195302.

[17] J. M. Liu, R. Yen, H. Kurz, N. Bloembergen, Applied Physics Letters, 39 (1981) 755.

[18] C. V. Shank, R. Yen, C. Hirlimann, Physical Review Letters, 5 (1983) 900.

[19] C. H. Crouch, J. E. Carey, J. M. Warrender, M. J. Aziz, E. Mazur, Applied Physics Letters, 84 (2004) 1850.

[20] M. A. Sheehy, L. Winston, J. E. Carey, C. M. Friend, E. Mazur, Chem. Mater., 17 (2005) 3582.

(Received: July 9, 2012, Accepted: February 15, 2013) 